\title{
General Theory of Predictive Dosimetry for Yttrium-90 Radioembolization to Sites Other than the Liver
}

\author{
Yung Hsiang Kao
}

Received: 22 July 2013/Accepted: 10 September 2013/Published online: 15 November 2013

(C) Springer Science+Business Media New York and the Cardiovascular and Interventional Radiological Society of Europe (CIRSE) 2013

\begin{abstract}
Modern advances in interventional radiology and nuclear medicine may now allow for safe and effective ${ }^{90} \mathrm{Y}$ radioembolization to sites other than the liver. A general theory of predictive dosimetry based on the MIRD schema is proposed, adapted from the original partition model for liver ${ }^{90} \mathrm{Y}$ radioembolization.
\end{abstract}

\section{Letter to the Editor}

In a recent publication, Ricke et al. [1] described the safe ${ }^{90} \mathrm{Y}$ radioembolization of lung tumors using resin microspheres. There also are recent reports of ${ }^{90} \mathrm{Y}$ radioembolization being safely performed to the kidney [2] and spleen [3]. However, as early as 1965, Blanchard et al. [4] showed that ${ }^{90} \mathrm{Y}$ radioembolization to sites other than the liver is risky, with potential for serious nontarget radiation toxicity. We are recently reminded that safe and effective ${ }^{90} \mathrm{Y}$ radioembolization is a combination of "technical success" and "clinical success" [5], the latter being a radiobiological function of tissue dose-response.

The aim of this Letter is to provide a dosimetric roadmap on predictive dosimetry for safe and effective ${ }^{90} \mathrm{Y}$ radioembolization to sites other than the liver. The concepts presented here are of a general nature, and user discretion is advised in accordance to the specific nature of each case, tissue radiobiology, dosimetric limitations, institutional expertise, equipment, and ethical standards.

\section{Y. H. Kao $(\bowtie)$}

Department of Nuclear Medicine, Austin Hospital, Level 1, Harold Stokes Building, 145 Studley Road, Heidelberg, VIC 3084, Australia

e-mail: yung.h.kao@gmail.com
Predictive dosimetry requires ${ }^{90} \mathrm{Y}$ microsphere biodistribution simulation using a suitable inert surrogate, e.g., ${ }^{99} \mathrm{~m}$ Tc macroaggregated albumin [6, 7], assessed by scintigraphy of the target arterial territory and relevant regionsat-risk. Conceptually, simulation scintigraphy should be regarded as a dosimetric map depicting the predicted final positions of all injected microspheres, where all possible flow-shunt-implantation distributions have irreversibly occurred.

\section{Dosimetric Assumptions}

(1) Only a single arterial territory is undergoing ${ }^{90} \mathrm{Y}$ radioembolization; (2) ${ }^{90} \mathrm{Y}$ microspheres are injected under identical technical and physiological circumstances as its surrogate (e.g., catheter position and type, hemodynamic status), as far as possible; (3) there are no more than three tissue compartments for dosimetry, i.e., tumor, nontumorous tissue, and lung (Fig. 1); (4) vascular stasis and microsphere reflux has not occurred at the conclusion of ${ }^{90} \mathrm{Y}$ radioembolization; (5) implanted tissue activity is proportional to scintigraphic counts, and its quantification is accurate; (6) there is no postimplantation redistribution of microspheres nor leeching of ${ }^{90} \mathrm{Y}$ activity; (7) there are no arteriovenous shunts from nontumorous tissue contributing to lung shunting of microspheres. If the user suspects that arteriovenous shunting may be occurring within nontumorous tissue (e.g., congenital vascular malformations, previous surgery, or trauma), the entire predictive dosimetry will revert in principle to the original "partition model" [8] as was developed for ${ }^{90} \mathrm{Y}$ radioembolization to the liver. This is because the original partition model accounts for the possibility of arteriovenous shunts occurring from nontumorous cirrhotic liver parenchyma. 


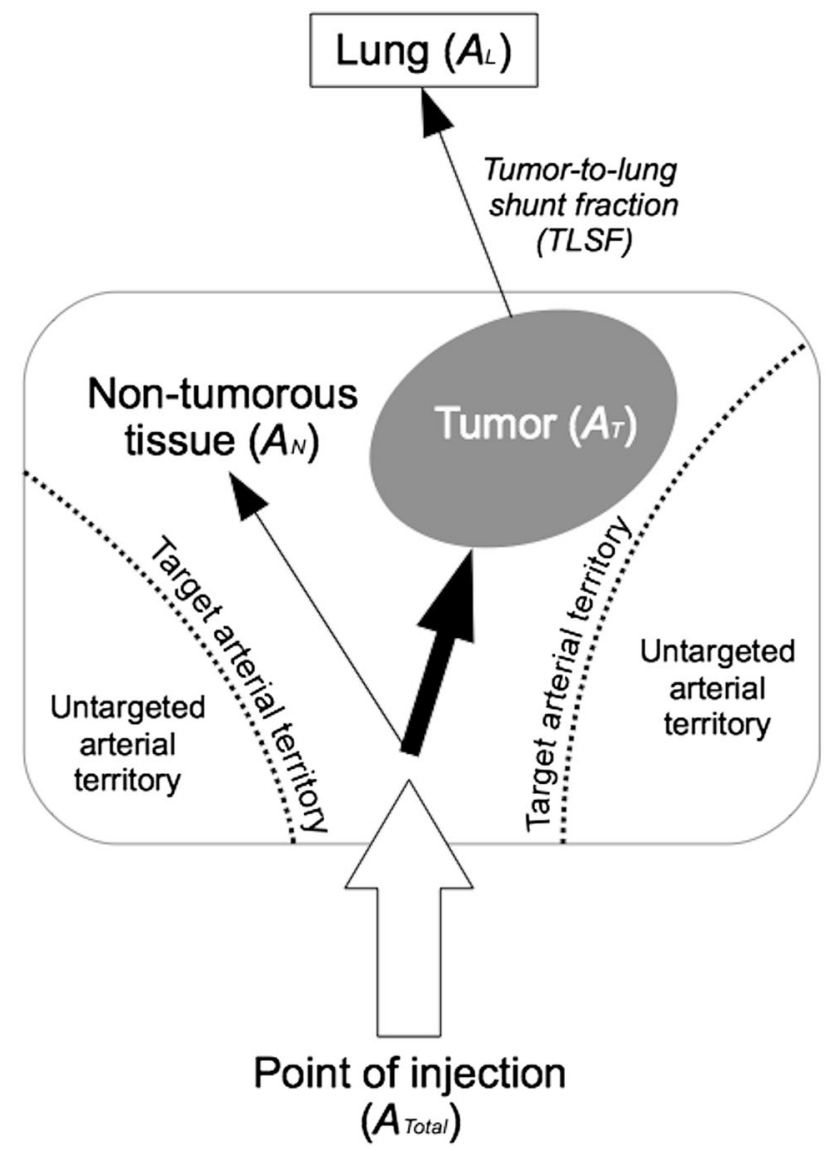

Fig. 1 Concept of tricompartmental MIRD predictive dosimetry involving the tumor, nontumorous tissue, and lung

\section{General Points}

(1) Minimum technology for simulation scintigraphy should be the single photon emission computed tomography with integrated CT (SPECT/CT); (2) no recommendations on desired absorbed doses are made here, as these depend on case-specific tissue radiobiology, technical factors and treatment intent (e.g., palliative, downstage, or cure); (3) dosimetric implications of the parallel-serial model in radiobiology, and the different radiobiological effects of ${ }^{90} \mathrm{Y}$ resin versus glass microspheres are not discussed here; (4) ${ }^{90} \mathrm{Y}$ glass microspheres, which have higher specific activity and lower microembolic potential, may have theoretical advantages in safety and efficacy over ${ }^{90} \mathrm{Y}$ resin microspheres in situations where vascular capacitance is anticipated to be low (e.g., intratumoral injection).

\section{General Dosimetric Theory}

Within a closed system where all injected activity is conserved, the desired total activity of ${ }^{90} \mathrm{Y}$ microspheres to be injected, $A_{\text {Total }}(\mathrm{GBq})$ is:
$A_{\text {Total }}=A_{\mathrm{T}}+A_{\mathrm{N}}+A_{\mathrm{L}}$

$A_{\mathrm{T}}$ is the total activity implanted within all tumor tissue, after shunting to nontumorous tissue and lung have occurred; $A_{\mathrm{N}}$ is the total activity implanted in all nontumorous tissue within the target arterial territory; $A_{\mathrm{L}}$ is the total activity implanted in lung due to tumor-to-lung shunting.

The tumor-to-lung shunt fraction (TLSF), is defined as:

$\mathrm{TLSF}=\frac{A_{\mathrm{L}}}{A_{\mathrm{T}}+A_{\mathrm{L}}}$

Equation 2 is approximated by $C_{\mathrm{L}} /\left(C_{\mathrm{T}}+C_{\mathrm{L}}\right)$, where $C_{\mathrm{L}}$ and $C_{\mathrm{T}}$ are the total counts in lung and all tumor tissue respectively, quantified by scintigraphy. The TLSF assumes no lung shunt contribution from nontumorous tissue, and is therefore conceptually different from the "liver-to-lung shunt fraction" defined by the original partition model [8].

The ratio of total activity in tumor to nontumorous tissue, $R$, is defined as:

$R=\frac{A_{\mathrm{T}}}{A_{\mathrm{N}}}$

Equation 3 is approximated by $C_{\mathrm{T}} / C_{\mathrm{N}}$, where $C_{\mathrm{N}}$ is the total counts in all nontumorous tissue quantified by scintigraphy. $R$ is a ratio of activities, not of mean radioconcentrations and, therefore, is conceptually different from the "tumor-to-normal liver ratio" defined by the original partition model [8].

The critical step in predictive dosimetry requires the user to decide an appropriate intended tissue mean absorbed dose to be delivered to any one of the three dosimetric compartments, i.e., tumor, nontumorous tissue, or lung. In this example, let us give a value to the intended tumor mean absorbed dose, $D_{\mathrm{T}}(\mathrm{Gy})$, defined as:

$D_{\mathrm{T}}=50\left(A_{\mathrm{T}} / m_{\mathrm{T}}\right)$ by Medical Internal Radiation Dose (MIRD) schema, where " 50 " is the approximate absorbed dose coefficient of $1 \mathrm{GBq}$ of ${ }^{90} \mathrm{Y}$ uniformly distributed throughout $1 \mathrm{~kg}$ of tissue [9], and $m_{\mathrm{T}}(\mathrm{kg})$ is the total mass of all tumors within the target arterial territory. Tissue mass $(m)$ may be estimated by CT volumetry and its mean radiographic density on a calibrated system, with attention to accurate delineation of volumes-of-interest. Therefore, $A_{\mathrm{T}}$ may be expressed as:

$A_{\mathrm{T}}=\frac{D_{\mathrm{T}} m_{\mathrm{T}}}{50}$

From Eqs. 3 and 4, the intended mean absorbed dose to non-tumorous tissue, $D_{\mathrm{N}}(\mathrm{Gy})$, of mass $m_{\mathrm{N}}(\mathrm{kg})$, is therefore:

$D_{\mathrm{N}}=\frac{D_{\mathrm{T}} m_{\mathrm{T}}}{R m_{\mathrm{N}}}$

From Eqs. 2 and 4, the intended mean absorbed dose to lung, $D_{\mathrm{L}}(\mathrm{Gy})$, of mass $m_{\mathrm{L}}(\mathrm{kg})$, is therefore: 


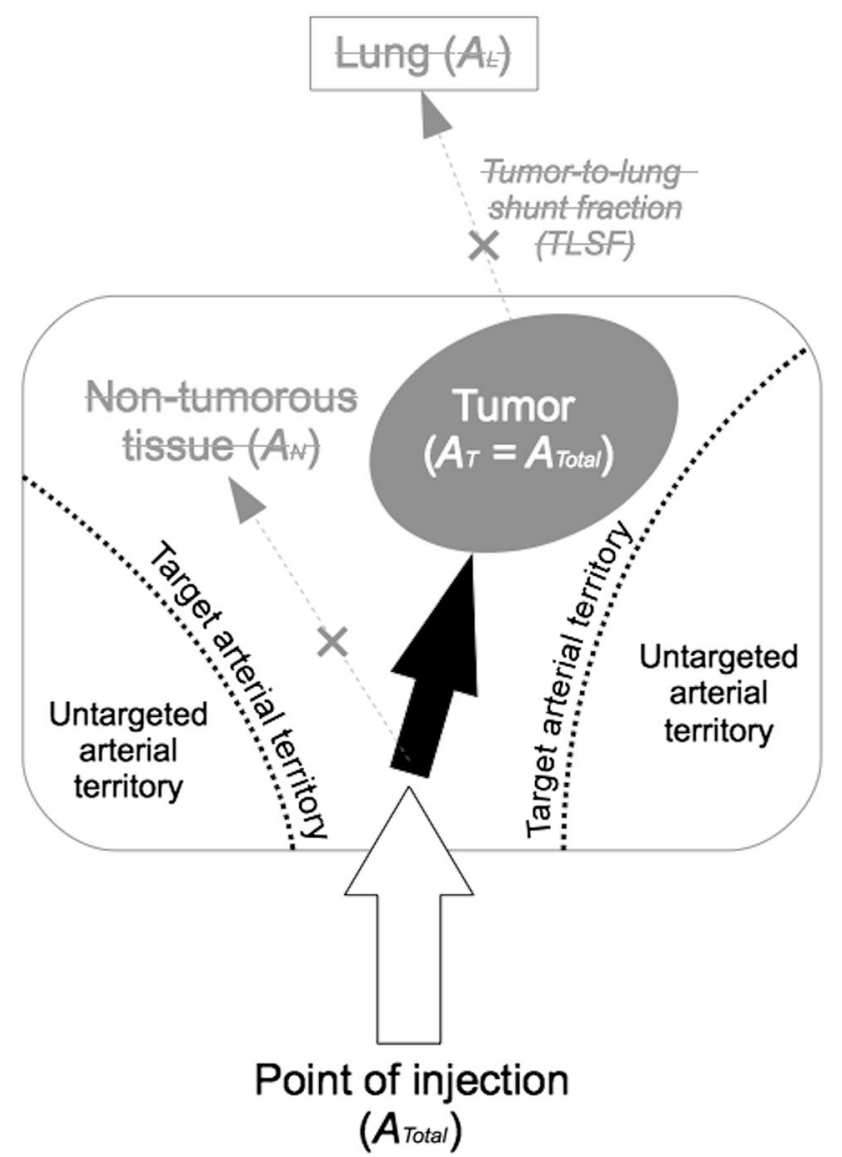

Fig. 2 The simplest dosimetric scenario where all injected ${ }^{90} \mathrm{Y}$ microspheres are completely implanted within tumor

$D_{\mathrm{L}}=\left(\frac{D_{\mathrm{T}} m_{\mathrm{T}}}{m_{\mathrm{L}}}\right)\left(\frac{\mathrm{TLSF}}{1-\mathrm{TLSF}}\right)$

Applying Eqs. 2, 3 and 4 into Eq. 1:

$A_{\text {Total }}=\frac{D_{\mathrm{T}} m_{\mathrm{T}}}{50}+\frac{D_{\mathrm{T}} m_{\mathrm{T}}}{50 R}+\left(\frac{D_{\mathrm{T}} m_{\mathrm{T}}}{50}\right)\left(\frac{\mathrm{TLSF}}{1-\mathrm{TLSF}}\right)$

In the simplest dosimetric scenario, all injected ${ }^{90} \mathrm{Y}$ microspheres are completely implanted within tumor, i.e., both $A_{\mathrm{N}}$ and TLSF are zero (Fig. 2). Therefore, Eq. 1 is reduced to simply:

$A_{\text {Total }}=A_{\mathrm{T}}=\frac{D_{\mathrm{T}} m_{\mathrm{T}}}{50}$

If nontumorous tissue is present within the target arterial territory (i.e., $A_{\mathrm{N}}>0$ ), but there is no tumor-to-lung shunting (i.e., TLSF $=0$ ), then Eq. 1 is reduced to:

$A_{\text {Total }}=A_{\mathrm{T}}+A_{\mathrm{N}}=\frac{D_{\mathrm{T}} m_{\mathrm{T}}}{50}+\frac{D_{\mathrm{T}} m_{\mathrm{T}}}{50 R}$

If tumor-to-lung shunting is present (i.e., TLSF $>0$ ), but there is no nontumorous tissue within the targeted arterial territory (i.e., $A_{N}=0$ ), then Eq. 1 is reduced to:

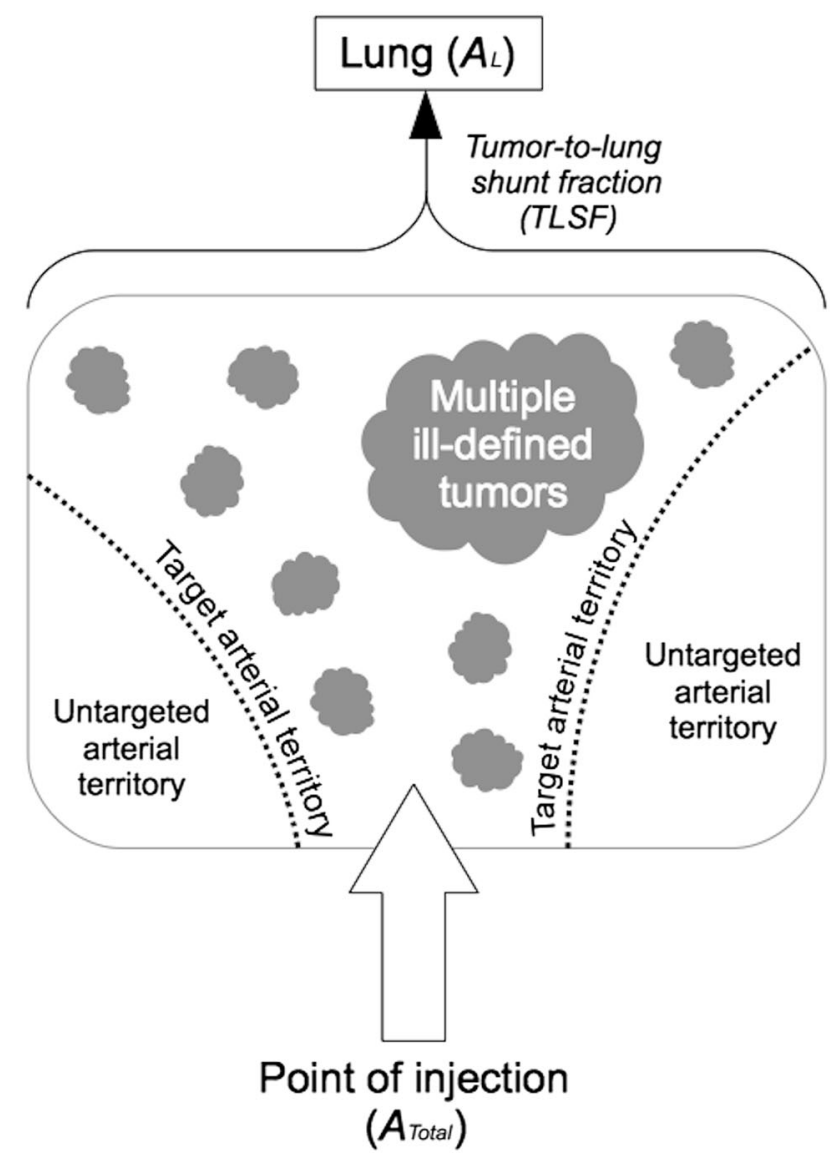

Fig. 3 For multiple, small or ill-defined tumor lesions, the entire target arterial territory may be conceptually regarded as "tumor" and treated by a single intended mean absorbed dose

$$
\begin{aligned}
A_{\text {Total }} & =A_{\mathrm{T}}+A_{\mathrm{L}} \\
& =\frac{D_{\mathrm{T}} m_{\mathrm{T}}}{50}+\left(\frac{D_{\mathrm{T}} m_{\mathrm{T}}}{50}\right)\left(\frac{\mathrm{TLSF}}{1-\mathrm{TLSF}}\right)
\end{aligned}
$$

In cases of multiple small or ill-defined tumor lesions which cannot be reliably quantified or delineated on imaging (e.g., diffuse lymphomatous infiltration), the user may choose to conceptually regard the entire target arterial territory as "tumor" (Fig. 3) and plan a single intended mean absorbed dose across the entire target arterial territory. Its dosimetry will be analogous to either Eqs. 5 or 6 , depending on the TLSF.

Technology and innovations that may improve the safety and effectiveness of ${ }^{90} \mathrm{Y}$ radioembolization to sites other than the liver are: (1) hybrid tomographic scintigraphy with integrated CT, i.e., SPECT/CT [7, 8] or PET/CT [10]; (2) antireflux catheters to minimize reflux of ${ }^{90} \mathrm{Y}$ microspheres into nontarget tissue [11]; (3) Voxel dosimetry and dose-volume histograms to overcome inherent limitations of dosimetric planning based on mean absorbed doses [12]; (4) multi-isotope surrogates to qualitatively and quantitatively assess microsphere biodistribution within 
multiple target arterial territories [13, 14]; (5) future possibility of combined external beam radiotherapy with ${ }^{90} \mathrm{Y}$ radioembolization.

Conflict of interest Yung Hsiang Kao had received previously research funding from Sirtex Medical Singapore.

\section{References}

1. Ricke J, Großer O, Amthauer H (2013) Y90-radioembolization of lung metastases via the bronchial artery: a report of 2 cases. Cardiovasc Intervent Radiol 36:1664-1669

2. Hamoui N, Gates VL, Gonzalez J, Lewandowski RJ, Salem R (2013) Radioembolization of renal cell carcinoma using yttrium90 microspheres. J Vasc Interv Radiol 24:298-300

3. Muylle K, Nguyen J, de Wind A et al (2013) Radioembolization of the spleen: a revisited approach for the treatment of malignant lymphomatous splenomegaly. Cardiovasc Intervent Radiol 36:1155-1160

4. Blanchard RJ, Lafave JW, Kim YS, Frye CS, Ritchie WP, Perry JF Jr (1965) Treatment of patients with advanced cancer utilizing Y90 microspheres. Cancer 18:375-380

5. Salem R, Lewandowski RJ, Gates VL et al (2011) Research reporting standards for radioembolization of hepatic malignancies. J Vasc Interv Radiol 22:265-278

6. Garin E, Lenoir L, Rolland Y et al (2012) Dosimetry based on 99mTc-macroaggregated albumin SPECT/CT accurately predicts tumor response and survival in hepatocellular carcinoma patients treated with ${ }^{90} \mathrm{Y}$-loaded glass microspheres: preliminary results. J Nucl Med 53:255-263
7. Kao YH, Hock Tan AE, Burgmans MC et al (2012) Image-guided personalized predictive dosimetry by artery-specific SPECT/CT partition modeling for safe and effective ${ }^{90} \mathrm{Y}$ radioembolization. J Nucl Med 53:559-566

8. Ho S, Lau WY, Leung TW et al (1996) Partition model for estimating radiation doses from yttrium-90 microspheres in treating hepatic tumours. Eur J Nucl Med 23:947-952

9. Kennedy A, Coldwell D, Sangro B, Wasan H, Salem R (2012) Radioembolization for the treatment of liver tumors general principles. Am J Clin Oncol 35:91-99

10. Selwyn RG, Avila-Rodriguez MA, Converse AK et al (2007) $18 \mathrm{~F}$-labeled resin microspheres as surrogates for ${ }^{90} \mathrm{Y}$ resin microspheres used in the treatment of hepatic tumors: a radiolabeling and PET validation study. Phys Med Biol 52:7397-7408

11. van den Hoven AF, Prince JF, Samim M et al (2013) Posttreatment PET-CT-confirmed intrahepatic radioembolization performed without coil embolization, by using the antireflux surefire infusion system. Cardiovasc Intervent Radiol, doi: 10.1007/ s00270-013-0674-3

12. Dieudonné A, Garin E, Laffont S et al (2011) Clinical feasibility of fast 3-dimensional dosimetry of the liver for treatment planning of hepatocellular carcinoma with ${ }^{90} \mathrm{Y}$-microspheres. J Nucl Med 52:1930-1937

13. Palmowski M, Goedicke A, Vogg A et al (2013) Simultaneous dual-isotope SPECT/CT with $99 \mathrm{mTc}$ - and 111In-labelled albumin microspheres in treatment planning for SIRT. Eur Radiol 23:3062-3070

14. Kao YH(2013) Personalized dosimetry applications of simultaneous multi-isotope albumin microspheres in pre-therapy planning for yttrium-90 radioembolization. http://www.european-radiology.org/ cms/website.php?id=/en/index/opinions/dosimetryapplications. htm. Accessed 11 Nov 2013 